\title{
EFFECT ASSESSMENT OF A COGNITIVE TRAINING PLATFORM IN HEALTHY OLDER ADULTS
}

\author{
Antonia Yaneva ${ }^{1}$, Nonka Mateva ${ }^{2}$
}

\begin{abstract}
Cognitive interventions, especially cognitive training, may improve cognitive functions in healthy older adults. Computerized cognitive training platforms offer several advantages over traditional programs for cognitive training and stimulation. The focus of this article is the methodology of the studies that apply a particular online training program. We investigate the effectiveness of several studies for cognitive training in healthy elderly people and evaluate reported outcomes and potential bias and what factors determine, influence or contribute to the positive or negative results. The post-intervention scores demonstrate that computerized cognitive training may enhance some cognitive functions and the overall cognitive status but there is need for additional research to prove its effectiveness.
\end{abstract}

JEL Classification Numbers: I120, C730; DOI: http://dx.doi.org/10.12955/cbup.v5.1066

UDC Classification: 614.4

Keywords: computerized cognitive training, assessment, older adults

\section{Introduction}

The ability of people to perform different cognitive tasks decreases with age. This may be due to normal aging or an indication of the onset of dementia or mild cognitive impairment (MCI). Cognitive decline leads to deterioration of the quality of life of these patients as well as difficulties or inabilities to perform activities of daily living. Currently, there is no cure for Alzheimer's disease and it is necessary to look for alternative methods to prevent and mitigate the consequences of the occurrence of this type of impairment. Many studies have shown that cognitive rehabilitation can prevent cognitive decline and even delay the onset of dementia (Papp et al., 2009). In one classification cognitive interventions are grouped into 3 categories - cognitive rehabilitation, cognitive stimulation and cognitive training (CT) (Clare et al., 2003). CT is defined by Martin et al. (2011) as "an intervention providing structured practice on tasks relevant to aspects of cognitive functioning, using standardized tasks" and "intended to address cognitive function and/or cognitive impairment directly. "Based on this and other similar definition, Gates \& Valenzuela (2010) determined that cognitive training is a specific form of cognitive intervention that aims to stimulate residual neuroplasticity in normal aging adults as well as those with mild cognitive impairment and Alzheimer's disease. In addition, they underline the increasing popularity of computer-based platforms that have the potential to produce more effective results on both general cognition and specific cognitive domains. Another systematic review (Kueider et al., 2012) investigated the efficacy of computer-based cognitive interventions and emphasized that they have significant advantages over traditional programs. Computer-based cognitive interventions offer the opportunity for personalized training taking into account the individual needs of the patient and allowing to focus only on those areas that need improvement. These programs are a much cheaper alternative that suggests much wider spreading among the elderly.

Cognitive training platforms

Recently, commercial cognitive training platforms have been becoming very popular. The most wellknown among them are Posit Science, CogniFit, Cogmed, Brain Age, My brain Trainer, Dakim and Lumosity. Despite the fact that they these programs are available, easy to use and could be adapted to different types of users, there is insufficient evidence for their clinical validity. A review of Shah et al. (2017) assesses the number and quality of studies that evaluate the empirical relevance of these platforms for cognitive function in healthy elderly (Shah et al., 2017). They group trials into three categories - I, II and III, level of evidence using PEDro scale (Physiotherapy Evidence Database scale) for randomized clinical trials. This scale consists of 11 criteria and helps to establish the validity of a randomized control trial (RCT) and if there are enough statistics that explain results. (Pedro et al., 1999). The authors have identified 2 programs with a level of evidence I - Posit Science and CogniFit.

\footnotetext{
${ }^{1}$ Faculty of Public Health, Medical University of Plovdiv, ayaneva@meduniversity-plovdiv.bg

${ }^{2}$ Faculty of Public Health, Medical University of Plovdiv, nmateva@meduniversity-plovdiv.bg
} 
CogniFit training program

CogniFit is a program designed specifically for the effective training of a variety of important cognitive skills. It covers a wide range of abilities, such as: visual search, time estimation, naming, categorization, visual short term memory, auditory short term memory, location memory, spatial orientation, planning, ability to inhibit planned action, speed of reaction and hand-eye coordination. Initially, CogniFit evaluates the starting point for each user and on the basis of this information it designs an individual training program. The training itself consists of tasks to be worked on for about fifteen to twenty minutes, three times a week. The software measures the person's progress and provides feedback on ongoing performance. (www.cognifit.com) (Breznitz)

\section{Objective}

The purpose of this review is to evaluate the effectiveness of cognitive training platform CogniFit in healthy older adults. Therefore, studies examining computer-based cognitive training are analyzed with regards to training effectiveness. Our goal is to assess the reported outcomes and potential bias and what factors determine, influence or contribute to the positive or negative results.

\section{Methodology}

Criteria for studies selection

This review focuses on RCTs for which adequate information was provided. The selected studies must be published and presented in a journal article. The participants must be over 50 years of age, healthy older adults with no diagnoses of dementia or Alzheimer's disease. The studies have to describe computer-based cognitive training interventions that targeted specific cognitive domains, or the overall cognitive status improvement. Control groups are defined as no training and the participants included receive no-cognitive training or stimulation. The studies must report participants' performance both in the beginning and at the end. The pre- and post-tests are evaluated regarding their relevance. To identify the appropriate studies, a search in healthcare databases was initiated using the following keywords: "cognitive training" or "cognitive stimulation" or "cognitive training platforms" or "CogniFit" and all terms are searched in the fields Title, Abstract and Keywords. The healthcare databases PubMed, Scopus, Web of Science and Springer were searched.

\section{Results}

We identified 29 articles that met the above-mentioned keywords criteria. Three of the studies were selected for inclusion in this review. These studies presented RCTs with healthy older adults with no diagnosis of dementia or MCI (Bellotti, 2014; Peretz et al., 2011; Stern et al., 2013). They included preand post-intervention tests of cognitive status and provided sufficient statistical data for the effect assessment of computerized cognitive training platform CogniFit. Over 26 studies were excluded because they focused on participants with multiple sclerosis, intellectual or developmental disabilities as well as older adults with insomnia (Haimov \& Shatil, 2013; Siberski et al., 2015; Shatil et al., 2010; Cimermanová \& Ram, 2013).

Design of the studies

All studies were randomized-controlled studies as per the inclusion criteria of the present paper. Two of the studies had one control group that used classic computer games or games that are especially designed for the study but have no cognitive training purpose (Bellotti, 2014, Peretz et al., 2011). One study included 4 groups - the first group was engaged in cognitive training, the second in mild aerobic training, the third in the combination of both and the fourth was not involved in any training but the patients included in it were engaged in book-reading activities (Stern et al., 2013).

\section{Patient recruitment}

The participants were healthy volunteers and some of them owned personal computers and the ability to use them. Individuals with corrected vision below 20/40 and impaired hearing as well as those with a history of clinical stroke or head trauma were excluded. Other exclusion criteria were alcohol dependence, any neurological diseases as well as the use of drug that could influence cognitive functions.

Test for baseline evaluations

Several neuropsychological tests were used for the evaluation of patients' performance before and after the training. Three of the studies used Mini-Mental Status Exam (MMSE) for the exclusion criteria. The 
test consists of 11 questions with a total score of 30 points. Results close to 0 indicate a significant cognitive deficit and those close to 30 - the absence of dementia or cognitive impairments (Folstein et al., 1975). Two other test, Trail Making Test (TMT) and Digit Span (DS), respectively, are used to measure executive functions such as complex visual-motor conceptual screening and test the working memory (Bellotti, 2014).

In addition to the above mentioned neuropsychological tests, the Schedule for the Evaluation of Individual Quality of Life (SEIQoL) was also used, which allows people to assess their quality of life by identifying the priority domains of life and also measure their satisfaction from each of them (O’Boye et al., 1993).

In order to evaluate the overall level of intelligence of the participants, the TONI-3 - Test of Non-Verbal Intelligence was used. This test is suitable for children over 6 years of age to adults 89 years and 11 months old and is a tool that assesses the cognitive function, capacity and abstract thinking of people with serious language problems. The test is well suited not only for English speakers but also for people who do not understand spoken or written English, either for cultural reasons or due to a trauma, a disease, or a disability (Brown, 2003).

An important part of the implementation of the CogniFit platform for cognitive training is the neurocognitive evaluation that is done before and after the intervention with the CogniFit Neuropsychological Evaluation. It consists of 15 tasks measuring multiple cognitive functions such as working memory, divided attention, planning and hand-eye coordination. The difficulty of the cognitive tasks involved in the cognitive training is adapted in accordance with the results from this assessment.

Duration of interventions

The training sessions lasted from eight weeks to 16 weeks, 3 times weekly, 20-30 min each session.

\section{Efficacy}

To evaluate the effect of the platform for cognitive training, mixed-effects models are used and Cohen's $d$ is calculated for the effect size between the two compared groups and within each group.

In the study of Peretz et al. (2013) the cognitive training group was compared with the control group that was engaged in playing classic computer games in order to evaluate eight cognitive domains: Focused attention, Sustained attention, Memory recognition, Memory recall, Visuospatial learning, Visuospatial working memory, Executive functions, Mental flexibility. The overall cognitive effect was presented in both groups. The within-group comparison shows that while in the group of cognitive training, an effect was observed in each of the 8 domains. The effect in the control group was only in 4 cognitive domains - Focused attention, Sustained attention, Memory recognition and Mental flexibility. Regarding the within-group differences, a borderline significance was found in the overall cognitive score. There was a highly significant improvement in the cognitive training group as compared to the control group in 3 domains: visual-spatial working memory, visual-spatial learning and focused attention. The effect was not significant in the 5 remaining domain, although the trend was in the same direction (Peretz et al., 2011).

The study of Bellotti (Bellotti, 2014) also showed improvement of working memory and executive function tasks for cognitive training group compared to the control group which completed a TV-based program of personally benefiting activities. A positive effect is also reported in the within-group comparison. The cognitive training group showed significant improvement on both the Digit Span Test and the Trail Making Test. Cohen's $d$ calculated for those abilities fell in the small to medium range -0 , 40 to 0,58 .

Cohen's $d$ is used when interpreting the effect of an intervention. Cohen's $d$ is the difference between the average scores on pre- and posttests firstly for the cognitive training group and then for the control group. Scoring was small or weak at $d=0.20$, medium at $d=0.5$ and large $d=0.8$ (Cohen, 1977).

In the study (Stern et al., 2013) there were 4 groups of participants. The effect of the training platform CogniFit was evaluated using The General Linear Model for Repeated Measures (SPSS v.18). In both groups, for cognitive training, and for cognitive training and physical exercises, a significant improvement in several verbal and nonverbal cognitive skills was reported: Hand-Eye Coordination, GVM (working memory and long-term memory), Speed of Information Processing, Visual Scanning, and Naming. Cohen's-, calculated for these improvements revealed medium-size ( $d=0.6$ or 0.7$)$ or large- 
size effects $(\mathrm{d}=0.8)$. In the cognitive training group significant improvements were found at the end of the study in terms Divided Attention, Avoiding Distractions, Hand-eye Co-ordination, Naming, Speed of Visual-Spatial Information Processing, Visual Scanning, and Global Visual Memory.

\section{Conclusion}

Computer-based platforms may provide objective data on patients' performance during the training. The studies included in this paper provide information and evidence that regular mental stimulation could result in improved cognitive ability. We suggest that relatively small improvements are due to duration of the study. But none of them reported if the acquired skills are sustainable and how long this effect lasts. Personalized and dynamically adapted cognitive training regarding individual capacity indicates a positive effect compared to computer games where the effect is haphazard, not so good or missing. In addition, combining cognitive training with physical activities would significantly increase the cognitive benefits compared to cognitive training and physical training separately.

\section{References}

Bellotti, F. (2014). Novel Television-Based Cognitive Training Improves Working Memory and Executive Function, 9(7). https://doi.org/10.1371/journal.pone.0101472

Breznitz, P. S. (n.d.). CogniFit Training: The key to cognitive vitality, 1-11.

Brown, L. (2003). Test of Nonverbal Intelligence. In Handbook of Nonverbal Assessment (pp. 191-221). Boston, MA: Springer US. https://doi.org/10.1007/978-1-4615-0153-4_10

Clare, L., Woods, B., Moniz Cook, E. D., Orrell, M., \& Spector, A. (2003). Cognitive rehabilitation and cognitive training for early-stage Alzheimer's disease and vascular dementia. In L. Clare (Ed.), Cochrane Database of Systematic Reviews (p. CD003260). Chichester, UK: John Wiley \& Sons, Ltd. https://doi.org/10.1002/14651858.CD003260

Cohen, J. (1977). Statistical power analysis for the behavioral sciences. Academic Press. Retrieved from http://www.sciencedirect.com/science/book/9780121790608

Folstein, M. F., Folstein, S. E., \& McHugh, P. R. (1975). "Mini-mental state". A practical method for grading the cognitive state of patients for the clinician. Journal of Psychiatric Research, 12(3), 189-198. https://doi.org/10.1016/00223956(75)90026-6

Gates, N., \& Valenzuela, M. (2010). Cognitive exercise and its role in cognitive function in older adults. Current Psychiatry Reports, 12(1), 20-27. https://doi.org/10.1007/s11920-009-0085-y

Haimov, I., \& Shatil, E. (2013). Cognitive Training Improves Sleep Quality and Cognitive Function among Older Adults with Insomnia. PLoS ONE, 8(4). https://doi.org/10.1371/journal.pone.0061390

Martin, M., Clare, L., Altgassen, A. M., Cameron, M. H., \& Zehnder, F. (2011). Cognition-based interventions for healthy older people and people with mild cognitive impairment. In M. Martin (Ed.), Cochrane Database of Systematic Reviews (p. CD006220). Chichester, UK: John Wiley \& Sons, Ltd. https://doi.org/10.1002/14651858.CD006220.pub2

Neuroscience, H., Cimermanová, D., \& Ram, I. (2013). Personalized cognitive training in unipolar and bipolar disorder : a study of cognitive functioning, 7(May), 1-10. https://doi.org/10.3389/fnhum.2013.00108

O’Boye, C. a, Browne, J., Hickey, A., McGee, H. M., \& Joyce, C. (1993). The Schedule for the Evaluation of Individual Quality of Life ( SEIQoL ): a Direct Weighting procedure for Quality of Life Domains ( SEIQoL-DW ) Administration Manual. Deparment of Psychology, Royal College of Surgeons in Ireland, 1-13.

Papp, K. V., Walsh, S. J., \& Snyder, P. J. (2009). Immediate and delayed effects of cognitive interventions in healthy elderly: A review of current literature and future directions. Alzheimer's \& Dementia, 5(1), 50-60. https://doi.org/10.1016/j.jalz.2008.10.008

Pedro, T., Ap, V., \& Delphi, T. (1999). PEDro scale. Physiotherapy Evidence Database, 2. https://doi.org/10.1016/S00049514(14)60281-6

Peretz, C., Korczyn, A. D., Shatil, E., Aharonson, V., Birnboim, S., \& Giladi, N. (2011). Computer-based, personalized cognitive training versus classical computer games: A randomized double-blind prospective trial of cognitive stimulation. Neuroepidemiology, 36(2). https://doi.org/10.1159/000323950

Shah, T. M., Weinborn, M., Verdile, G., Sohrabi, H. R., \& Martins, R. N. (2017). Enhancing Cognitive Functioning in Healthly Older Adults: a Systematic Review of the Clinical Significance of Commercially Available Computerized Cognitive Training in Preventing Cognitive Decline. Neuropsychology Review. https://doi.org/10.1007/s11065-016-9338-9

Shatil, E., Metzer, A., Horvitz, O., \& Miller, A. (2010). Home-based personalized cognitive training in MS patients: A study of adherence and cognitive performance. NeuroRehabilitation, 26(2). https://doi.org/10.3233/NRE-2010-0546

Siberski, J., Shatil, E., Siberski, C., Eckroth-Bucher, M., French, A., Horton, S., ... Rouse, P. (2015). Computer-based cognitive training for individuals with intellectual and developmental disabilities: Pilot study. American Journal of Alzheimer's Disease and Other Dementias, 30(1). https://doi.org/10.1177/1533317514539376

Stern, M., College, A., \& Valley, J. (2013). Does combined cognitive training and physical activity training enhance cognitive abilities more than either alone? A four-condition randomized controlled trial among healthy older adults, 5(March), 1-12. https://doi.org/10.3389/fnagi.2013.00008 